\title{
Low-Fluence Electron Yields of Highly Insulating Materials
}

\author{
Ryan Hoffmann, JR. Dennison, C.D. Thomson and J. Albretsen
}

\begin{abstract}
Electron-induced electron yields of high-resistivity, high-yield materials - ceramic polycrystalline aluminum oxide and the polymer polyimide (Kapton $\mathrm{HN}$ ), - were made by using a low-fluence, pulsed incident electron beam and charge neutralization electron source to minimize charge accumulation. Large changes in energy-dependent total yield curves and yield decay curves were observed, even for incident electron fluences of $<3 \mathrm{fC} / \mathrm{mm}^{2}$. The evolution of the electron yield as charge accumulates in the material is modeled in terms of electron recapture based on an extended Chung-Everhart model of the electron emission spectrum. This model is used to explain anomalies measured in highly insulating, high-yield materials, and to provide a method for determining the limiting yield spectra of uncharged dielectrics. Relevance of these results to spacecraft charging is also discussed.
\end{abstract}

Index Terms — charging, electron, emission, dielectrics

\section{INTRODUCTION}

$\mathrm{T}$ he central theme of spacecraft charging is how spacecraft interact with the plasma environment to cause charging. Spacecraft materials accumulate negative or positive charge and adopt potentials in response to interactions with the plasma environment. A material's electron emission, or electron yield, determines how much net charge accumulates in spacecraft components in response to incident electron, ion, and photon fluxes. Due to their high mobility, incident electrons play a more significant role in electron yield response and in resulting spacecraft charging than do positively charged ions. The electron emission properties of electrically-insulating materials are central to modeling spacecraft charging, as a function of incident electron energy since insulating materials generally exhibit higher yields than conducting materials, and accumulated charge cannot be easily dissipated. Furthermore, electron emission in insulators is complicated by the fact that the yield itself is affected by

Manuscript received February 1, 2008. This study was partially funded by the NASA Solar Probe Mission through the Johns Hopkins University Applied Physics Laboratory and by the NASA Space Environments and Effects program.

Ryan Hoffmann is a graduate student with the Department of Physics, Utah State University, Logan, UT 84322 USA, (Phone: 435-563-6206; e-mail: Ryanphyx@gmail.com).

JR Dennison is a professor in the Department of Physics, Utah State University, Logan, UT 84322 USA (e-mail: JR.Dennison@usu.edu).

C.D. Thomson is with ATK Launch Systems, Clearfield, UT 84016 USA, (e-mail: Clint.Thomson@atk.com).

J. Albretsen is an undergraduate student with the Department of Physics, Utah State University, Logan, UT 84322 USA, (e-mail: jenalbretsen@gmail.com). accumulated surface and bulk charge [1]. In order to more accurately describe the electron-induced charging behavior of insulators used on spacecraft, we have developed a model that quantifies the response of the electron yield as a function of accumulated charge and material surface potential.

In this paper, we present a study of the change in electron-induced electron yield that result from the buildup of internal charge distributions due to incident and emitted electron fluxes. Specifically, we look at how charge buildup in highly charged insulating materials affects these fluxes. First, we show an evolution of total and secondary yield results over a broad range of incident energies in response to accumulated charge for Kapton HN and aluminum oxide. Quantifiable changes in yields are observed in response to fluences less than $3 \mathrm{fC} / \mathrm{mm}^{2}$. We then present a model for the evolution of electron yields as a result of surface charging. This expression is derived from the physics based model for the emission spectrum of secondary electrons developed by Chung and Everhart [2], [3]. This model is fit to measured data to provide electron yields as a function of both incident electron energy and fluence. Using the double dynamic layer model for the internal charge distribution developed in response to incident charge, we present a model for the electron emission yield as a function of incident charge or equivalently surface potential[4]-[6]. Finally, we present an estimate of the "intrinsic" electron yield curve extrapolated to a minimal accumulated internal charge distribution.

\section{THEORETICAL MODEL}

\section{A. Electron Yield and Emission Spectra}

1) Electron Induced Electron Yield

The total yield, $\sigma$, is the ratio of emitted electron flux to incident electron flux. By convention, the secondary electron (SE) yield, $\delta\left(E_{o}\right)$, is the ratio for emitted electrons with energy $<50 \mathrm{eV}$ and the backscattered electron (BSE) yield, $\eta\left(E_{o}\right)$, is the ratio for emitted electrons with energy $>50 \mathrm{eV}$. An electron yield curve on gold shows the yield as a function of incident electron energy (see Fig. 1a). The total yield curve can be characterized in terms of five parameters [7]: (i and ii) the first and second crossover energies, $E_{1}$ and $E_{2}$, occur when the total yield is equal to unity and no net charge is deposited; (iii and iv) the yield peak, $\sigma_{\max }$, is the maximum yield and occurs between the crossover energies at $E_{\max }$ (the maximum yield is typically found between $200<E_{\max }<1000 \mathrm{eV}$ ); and (v) the rate at which the yield approaches the asymptotic limit, $\sigma \rightarrow 0$, with increasing beam energy, $E_{o} \rightarrow \infty$. 
The electron emission properties of conductors are relatively easy to measure, because emitted electrons are rapidly replaced by connecting the material to ground [8], [9]. However, yield measurements on dielectrics are more difficult because of the inability to ground the dielectric and the resulting response of the yield to charge accumulation [10], [11]. Accumulated charge in insulators interacts with both incident and emitted charged particles through Coulomb interactions and affects electron emission in all three stages of emission models as reviewed in Thomson [10]. Surface potentials resulting from the accumulated charge can influence yields by altering incident (or landing) energies, by affecting the escape energies of secondary electrons (SEs) and backscattered electrons (BSE) or by reattracting low energy SE to a positively charged surface.

For example, Fig. 3a shows a very low fluence yield curve taken on polycrystalline aluminum oxide, a highly charging material with a combination of high yield and high resistivity. It is easy to see that charging plays a significant role in the shape of the yield curve. Despite using very small incident fluences, the depressed yield curve between the observed peaks suggests that significant positive charging is nonetheless being induced. This, in turn, lowers the yield by reattracting some fraction of the SE. This dual-peak behavior is only seen in our system on insulators with $\sigma_{\max }>\sim 5$. This is due to the fact that higher yields require less incident flux to induce charging. In addition, highly resistive materials do not dissipate significant charge on the time scale of the measurement.

\section{2) Electron Emission Spectra Related to Total Yield}

A review of the electron emission spectra illuminates how charge accumulation affects the yield. Chung provides a useful model for the electron emission spectra, which expresses the energy distribution of the number of emitted SE per unit energy, $N(E)$, in terms of the work function for metals, $\varphi[2]$, [3] [9]. In the case of insulators, the literature supports a simple substitution of the electron affinity, $\chi$, for the work function [10].

$$
\frac{d N\left(E ; E_{o}\right)}{d E}=\frac{k}{E_{o}} \frac{E}{(E+\chi)^{4}}
$$

where $\mathrm{E}$ is the SE emission energy and $\mathrm{k}$ is a materialdependent proportionality constant. The SE yield in terms of $\mathrm{N}(\mathrm{E})$ is given by

$$
\int_{0 e V}^{50 e V} \frac{d N\left(E ; E_{o}\right)}{d E} d E=\delta_{o}\left(E_{o}\right)+\eta_{o}-1=\sigma_{o}\left(E_{o}\right)-1 .
$$

Here

$$
N \equiv \frac{N_{\text {emitted }}-N_{\text {incident }}}{N_{\text {incident }}}
$$

is the net number of emitted electrons, leading to the 1 on the right side of (2). Measured emission spectra for Au are shown in Fig 1b, along with a fit based on the Chung-Everhart model. Between the total-yield crossover energies, $E_{1}$ and $E_{2}$, the magnitude of insulator charging is positive (since the total yield is greater than one), and due to the reattraction of low energy electrons, the insulator attains a steady-state surface potential of just a few volts positive. This positive charging increases the insulator surface potential barrier by an amount $e V_{s}$, where $V_{s}$ is the positive surface potential. Hence, the resulting secondary electron yield emitted from a positively charged specimen can be expressed as an integral of the uncharged spectrum (taken at the same incident energy) with the integration limits extending from the positive surface potential up to the arbitrary $50 \mathrm{eV}$ limit of SE energy [12], [13]. $\eta\left(E_{o}\right)$ is assumed to be unaffected by the built up potential in the following discussion:

$$
\int_{e V_{s}}^{50 e V} \frac{d N\left(E ; E_{o}\right)}{d E} d E=\delta\left(E_{o} ; V_{s}\right)+\eta_{o}\left(E_{o}\right)-1=\sigma\left(E_{o} ; V_{s}\right)-1 .
$$

This integral can be solved analytically by direct substitution of (1) into (3) as

$\sigma\left(E_{o} ; V_{s}\right)-1=\frac{k}{6 E_{o}}\left[h\left(e V_{s} ; \chi\right)-h(50 e V ; \chi)\right]$

where

$h(\alpha, \chi) \equiv \frac{3 \alpha+\chi}{(\alpha+\chi)^{3}}$

and $\alpha$ is a dummy variable.

This is illustrated in Fig. 1b, where the positive surface charging inhibits the escape of lower-energy SE's, thus suppressing the lower-energy portion of the SE spectrum (represented by the shaded area in Fig. 1b). Consequently, only the unshaded area of the electron energy spectrum (above $e V_{s}$ ) contributes to the charged electron yield. It follows that the fraction of the SE yield escaping the surface is

$H\left(V_{s} ; \chi\right) \equiv \frac{\sigma\left(E_{o} ; V_{s}, \chi\right)-1}{\sigma_{o}\left(E_{o}\right)-1}=\frac{h\left(e V_{s} ; \chi\right)-h(0 e V ; \chi)}{h(50 e V ; \chi)-h(0 e V ; \chi)}$

As illustrated in Fig. 1c, (4), gives the fraction of the generated SE that have enough energy to overcome the surface potential and contribute to the yield. For charged insulators, this is the fraction of secondary electrons that escape the intrinsic electron affinity and the positive surface potential created by incident charge. Using (4), to solve (3), for the secondary yield as a function of surface potential, $V_{s}$, we obtain

$$
\begin{aligned}
& \delta\left(E_{o}, V_{s} ; \chi\right)=\sigma\left(E_{o}, V_{s} ; \chi\right)-\eta_{o}\left(E_{o}\right) \\
& =\left[\sigma_{o}\left(E_{o}\right)-1\right] \bullet H\left(V_{s} ; \chi\right)-\eta_{o}\left(E_{o}\right)+1
\end{aligned}
$$


$\sigma_{o}$ is the uncharged total yield; in practice this becomes the minimally charged yield and is used as a fitting parameter. With $\chi$ (the electron affinity) representing an intrinsic material property, (5), is a two parameter analytic expression for the yield in response to surface potential. Measurements of $\sigma\left(V_{s}, E_{o} ; \chi\right)$ at a given $E_{o}$ as a function of $V_{s}$ have been termed electron yield decay. To proceed we need to develop a specific expression for the surface potential $V_{s}$ as a function of incident charge $Q_{o}$, as it appears in the lower limit of the integral in (3).

\section{B. Charge Distribution in Insulators}

Let us consider a succession of more sophisticated charge distributions. For the purposes of this study we will focus only on the incident electron energies between the crossover energies (i.e., the positive charging regime). For biased conducting materials, the charge resides near the surface in accordance with Gauss' law. For ideal insulators, one assumes that incident or primary electrons (PE) do not move appreciable distances within the material and that the SE charge distribution is the same as the production profile. The simplest model of charge distribution in an insulator is that all incident charge is deposited in a thin layer at a depth equal to the penetration depth of the primary electron, $R\left(E_{o}\right)$. This follows from the Bethe approximation for SE production used in the Sternglass formulation of the yield formula [14], [15].

To first order, we can model the charge deposition as a single infinite charge layer at the surface of a sample of thickness $D$. Using a simple parallel plate capacitor model with the net total electron yield dependence included gives

$V_{s}=\frac{Q_{o}(\sigma-1) D}{\varepsilon_{o} \varepsilon_{r} A_{o}}$

As expected, for (6), $V_{s}$ is positive (negative) for $\sigma$ greater (less than) unity and in the limit were $\sigma \rightarrow 1$ no charging occurs. While this model provides a useful first order approximation for the surface potential it is rather simplistic in its treatment of the internal charge distribution. Finite resistivity allows redistribution of charge within the insulator, leading to more complicated internal charge distributions [16]. Previous models of insulators have shown that the internal charge distributions (both evolving distributions as well as static charge distributions), resulting from incident electron irradiation, form multiple alternating positive and negative charge layers [6], [7], [17]-[20]. Measurements of internal charge distributions of thin-film insulators confirm the general nature of these distributions [21]-[23]. However, the spatial and charge-polarity configurations of these layers can be complex and difficult to predict; the distributions can depend on a number of factors that include the magnitude of electron yield, electron yield crossover energies (particularly $E_{2}$ ), material conductivity (both dark current and radiation-induced conductivity), dielectric strength, electron trapping and detrapping rates, incident electron penetration depths, mean SE escape depths, and incident electron fluxes and energies.
The combination of these layers is what defines the overall magnitude of the surface potential. Thomson provides a useful review of the literature on charge distributions within insulators, with application to electron emission from insulators [10].

Between the crossover energies, incident electron penetration is only somewhat larger than the SE escape depth, a double-charge distribution (positive-negative) is formed where the positively charged region, from SE depletion, occurs between the surface and $\lambda_{S E}$ and a negatively charged region, from embedded incident electrons, occurs between the surface and $R$ (see Fig. 2). The electric field from the negative charge again retards further incident electron penetration and acts to drive more low-energy SE from the sample, thereby enhancing the positive charge region [11], [13]. The electric field from the positive charge region, in turn, acts to reattract the lowest energy SE emitted from the surface (gray region in Fig. 1b), thereby establishing a shallow negative surface charge region. For this charging scenario, the dynamic double layer model (DDLM) has been presented in the literature to predict ensuing internal electric fields and potentials [17], [18,] [24]. For the DDLM charge distribution deposited over a thickness, the surface potential can be approximated assuming a parallel-pate capacitor geometry with total incident charge $Q_{o}$ as [10], [19]

$V_{S}=\frac{Q_{o} D}{\varepsilon_{o} \varepsilon_{r} A_{o}}\left[(\sigma-1)-\sigma \frac{\lambda_{s e}}{2 D}-\frac{R}{2 D}\right]$.

The first term is from the net charge distribution of magnitude $Q_{o}(\sigma-1)$ given by (6), the term involving $\lambda_{s e}$ is for the positive charge distribution of magnitude $Q_{o} \sigma$ from SE emission, and the term involving $\mathrm{R}$ is for the imbedded $\mathrm{PE}$ distribution of magnitude $Q_{o}$. The thin-film capacitor geometry is a reasonable approximation since the charge deposition area $A_{o}$, which is given by the electron beam radius $R_{\text {beam }}$, is much greater than $D, R$, and $\lambda_{S E}$ (for studies reported here, $R_{\text {beam }}$ was on the order of $1.5 \mathrm{~mm}$, whereas insulator thicknesses ranged from $5 \mu \mathrm{m}$ to $1 \mathrm{~mm}$ ). Furthermore, it can be seen that the first term in (7), dominates if the insulator thickness $D$ is much greater than $R$ or $\lambda_{S E}$ ( $R$ did not exceed $\sim 1 \mu \mathrm{m}$ for the incident energies reported here); this approximation is equivalent to assuming a uniform charge distribution, as given in (6). Notice that $V_{s}$ is also a function of the total yield $\sigma\left(Q_{o}\right)$, which itself is dependant on incident charge.

\section{Response of Total Yield to Evolving Surface Potential}

We can now combine our expression for the electron yield in terms of the Chung-Everhart model of electron emission (2)-(4), with a model of the surface voltage in terms of incident charge from the DDLM model (7), to derive a model for the evolution of the yield in response to positive surface potential. Both of these component models are physics based and have been experimentally validated. In order to proceed, and combine these two expression, we need to make several assumptions. 
1) The energy distribution of emitted electrons given by (1), does not change shape with charge accumulation, but only changes amplitude and peak position. Experimental evidence for both biased conductors and charged insulators and the theoretical development by Chung [2], [3], suggest this is a reasonable assumption [11], [12].

2) The BSE yield is assumed to be unaffected by the positive surface potential developing on the sample. This is reasonable as long as the incident energies are much greater than the surface potential. In the positive charging regime this is true because the surface potential is never more that about $+20 \mathrm{eV}$, and usually only a few $\mathrm{eV}$. Further, we assume that the BSE yield is independent of incident electron energy, that is $\eta\left(E_{o}\right) \rightarrow \eta_{o}$.

3) No significant charge is leaking though the sample to ground on the time scale of our measurements. This is reasonable, given the bulk resistivities of $10^{17}$ to $10^{19} \Omega$ $\mathrm{cm}$ for materials studied here that correspond to charge decay times of 20 to 50 days, whereas the electron emission measurements take only seconds. To explicitly include charge dissipation, the surface voltage distribution of (6) or (7) must become time dependant, with a time dependant expression for incident charge $Q(t)$ and subtraction of a new term proportional to the rate of change dissipation that reflects the material conductivity and dielectric constant.

While these assumptions make the derivation possible we still encounter considerable difficulty when merging these two models due to the limit of integration for (3). This is due to the fact that $V_{s}$ is itself a function of the total yield $\sigma$. In order to get an expression for measured electron yield decay data $\sigma$ verses accumulated incident charge (or equivalently surface potential) one need only plot $\sigma\left(E_{o}, V_{s}\right)$ verses $Q_{o}\left(V_{s}\right)$ with either $V_{s}$ or $Q_{o}$ defined implicitly.

\section{EXPERIMENT}

\section{A. Instrumentation and Methods}

We briefly describe the instrumentation used at Utah State University (USU) to study electron emission from insulators [8]. Electron emission measurements are performed in an ultra-high vacuum chamber (base pressure $<10^{-9}$ Torr) to minimize surface contamination that can substantially affect emission properties [24], [25]. Electron sources provide electron energy ranges from $\sim 50 \mathrm{eV}$ to $\sim 30 \mathrm{keV}$ and incident electron currents (1-100 nA) with pulsing capabilities ranging from $10 \mathrm{~ns}$ to continuous emission [8]-[10]. A hemispherical detector features an aperture for incident electron/ion admission and a fully-encased hemispherical collector for full capture of emitted electrons with a retarding-field analyzer grid system for emitted-electron energy discrimination [9][11]. A sample stage holds 11 samples that can be positioned in front of various sources and detectors and is detachable for rapid sample exchange.

A DC method with a continuous, low-current beam of electrons is used to measure electron emission from conducting samples. Charge added to or removed from a conductor via electron emission can be rapidly replaced by connecting the sample to ground [8], [9]. Reviews of methods used by previous investigators to study insulator emission are found in Thomson [10] and reference [26]. The fully encased hemispherical grid retarding field detector facilitates high accuracy measurements of absolute yields, on the order of $\pm 2 \%$ for conducting samples. It also allows the application of bias to each of the discrete elements of the detector. These biases allow for the discrimination of secondary and backscatter electrons and measurement of electron emission spectra. Finally, the individually biased elements of the detector allow for extensive instrument characterization. For conductor measurements, a continuous incident beam is shone on the sample and the currents on all the elements are measured using electrometers. This allows measurement of the total yield. A $-50 \mathrm{~V}$ bias is then applied to the discriminating grid to allow only the BSE to reach the detector, thus giving the BSE yield. The secondary yield is then the difference of these two measurements. Electron emission spectra are obtained using the same apparatus with the discrimination grid stepping through a range of voltages.

\section{1) Insulators Electron Yield Measurement Techniques}

The system at USU to measure electron emission from insulators uses the same full encased hemispherical grid retarding field detector in concert with methods to control the deposition and neutralization of charge [10], [11], [26], [27]. Typically, charge deposition is minimized by using a low current beam ( 5-30 nA) focused on a sample area of $\sim 7 \mathrm{~mm}^{2}$ that is delivered in short pulses of $\sim 5 \mu$ sec. Each such pulse contains $\sim 10^{6}$ electrons $/ \mathrm{mm}^{2}$. The pulsed system uses custom detection electronics with fast (1-2 $\mu$ s rise time) sensitive $\left(10^{7}\right.$ V/A) low noise $(<100 \mathrm{pA})$ ammeters [10], [27]. Charge dissipation techniques include a low energy $(\sim 1-10 \mathrm{eV})$ electron flood gun for direct neutralization of positively charged surfaces and a variety of visible and UV light source for neutralization of negatively charged surfaces through the photoelectric effect [10], [11]. Sample heating to $\sim 50-100{ }^{\circ} \mathrm{C}$ has also been used for dissipation of buried charge by thermally increasing the sample conductivity.

To measure points on the yield curves at a particular energy, a series of 10 to $50 \sim 5 \mu$ s pulses at constant incident energy are measured with 5-10 sec of neutralization between each pulse, using both low energy electron and visible-ultraviolet flooding. The neutralization technique has been experimentally verified to be an effective method for discharging positive surface potential (see Fig. 3b).A similar series of pulses at fixed incident energy, taken without neutralization, constitute yield decay curves.

\section{B. Electron Emission Measurements on Insulators}

Using the method described above we have been able to measure yields on insulators with errors of $\pm 5 \%$. This method has been used to measure insulators with modest resistivity $\left(\sim 10^{15} \Omega\right.$-cm) and modest emission $\left(\sigma_{\max } \sim 4\right)$. It has also been 
used for insulators such as Kapton with high resistivity $\left(\sim 10^{19}\right.$ $\Omega$-cm) and modest emission ( $\sigma_{\max } \sim 3$ ). As engineering demands become more extreme, so do the demands on the materials, forcing the use of insulators with both higher resistivity and a higher yield. One such material is polycrystalline aluminum oxide with a resistivity of $\sim 10^{17} \Omega$ $\mathrm{cm}$ and a $\sigma_{\max }$ of $\sim 7$. While our methods are effective at dissipating charge, we are limited by how small the incident fluence can be, before the emission signal is lost in the noise. In insulators with modest yield, the incident pulse does not produce enough secondary electrons to appreciably charge the sample; however, in high yield insulators the incident pulse does. This is evident in Fig. 3a: at energies that have a low yield, in the leading and trailing edge of the yield peak, we see little evidence of charging, while in the middle where the yield should be the highest, we see significant charging. Severe undissipated positive charging in the peak energy range causes the yield to be suppressed toward unity, as we would expect for positive surface potentials.

To verify the effectiveness of the pulse neutralization method described above, a long series of pulsed measurements at a fixed energy and fluence were taken to look for any change in the yield that would indicate residual potential building up from pulse to pulse. The data presented in Fig. 3b do not show any long term change in the total yield. There is a slight upward trend with an $7 \%$ increase in the yield over the full length of the experiment with $~ 500$ pulses and a total incident dose of $\sim 20 \mathrm{pC} / \mathrm{mm}^{2}$; this is attributed to instrumental drift over the 4 hour duration of the experiment.

\section{1) Yield Decay Curves}

By measuring a sequence of yields with the method described above, without discharging the material between pulses, we generate a yield decay curve. This allows more and more charge to accumulate within the sample with each incident pulse, thus reattracting more secondary electrons until the yield approaches unity. This is shown in Fig. 4a for aluminum oxide. From these data we see a $23 \%$ change in the yield over 50 pulses of $\sim 10^{6}$ electrons per pulse, as compared to a $<1 \%$ change when using neutralization in between incident pulses.

\section{2) Yield Dose Decay Curves}

Flooding the sample with low-energy electrons between each incident pulse stops residual surface potential build up from affecting the yield from pulse to pulse. However, the question still remains, whether a single incident pulse contains sufficient electrons to induce significant charging. In other words, is the incident pulse inducing enough charge within the duration of the pulse to appreciably affect the yield? The results of a low fluence measurement of the yield curve seen in Fig. 3a, suggests that a single pulse is affecting the yield that results within its duration. To verify this, the yield was measured as the fluence per pulse was varied (Fig. 4b). In this case the potential was not allowed to accumulate from pulse to pulse as in a typical decay curve as shown in Fig. 4a (material discharging was employed between each pulse). Instead, the beam diameter and incident energy were kept constant and only the number of incident electrons per pulse was varied. It is evident that as the incident fluence decreases, the yield continues to rise (consistent with lower positive surface potential buildup). Eventually we reach an instrumental limit and cannot make yield measurements at lower fluences. Work is in progress to lower the noise threshold in our system to enable measurements of the limiting case of minimally charged yield. In addition to this, an apparatus to measure the in situ surface charging is being developed. It must be mentioned that as a result of these findings we are forced to reevaluate much of the measured insulator yield data reported in the literature, since in past studies, the incident charge per pulse has not been a carefully monitored measurement parameter. The prevailing carelessness in regulating this measurement parameter has most likely resulted from more traditional conductor yield measurements, where the electron yields are considered to be independent of incident charge levels, and only depend on incident electron energies. However, as we have shown, incident charge doses as small as $1-5 \mathrm{pC} / \mathrm{mm} 2$ can significantly alter electron yields, especially for high-yield insulators.

\section{Reconstruction of Uncharged Yield Curve}

Measuring the yield for a minimally charged insulator may be possible if the noise in the system can be sufficiently reduced. In the mean time, we propose a method for turning charging to our advantage. In section II, we developed a method for determining the dependence of the yield on surface potential. Equation (7) provides a model to calculate surface potential from the accumulated incident charge density. Equations (5) and (7) with $V_{s}$ as an implicit variable allow calculation of yield as a function of cumulative charge, that is, the yield decay curves. In practice the lower integration limit in (2) needs to reflect the average residual charge accumulated on the surface during the first pulse. As an approximation, we use the yield measured with this first pulse as $\sigma_{o}\left(E_{o}\right)$ in (5). This now provides an expression for the yield as a function of surface potential.

Decay curves were measured over a spectrum of 21 incident energies ranging from $200 \mathrm{eV}$ to $5000 \mathrm{eV}$ and fit with (5). A representative current with incident energy of $200 \mathrm{eV}$ for aluminum oxide is shown in Fig. 4a. (This method of yield measurement is invalid at energies below $200 \mathrm{eV}$ because we can no longer make the assumption that the BSE are not affected by surface potential). We can then predict yield curves as a function of incident energy by determining the yield at a specific cumulative incident charge resulting from the fits to the measured decay curves.

While this method is very time intensive, it can provide a great wealth of information. We can extrapolate these decay curve fits back to a zero surface potential to generate the "intrinsic" yield curve shown in green on Fig. 3a. When compared to the traditional yield curve measurements (blue data in 3a) described in section III.A, we see that this resolves charging difficulties, predicts a much higher $\sigma_{\max }$, and 
eliminates the double peak behavior. In addition, Fig. 4c shows the yield curves predicted at several representative surface potentials; we see that, as the potential increases, we start to see the emergence of the dual-peak behavior observed in the traditional low-fluence pulsed method of yield measurement and that at higher surface potential the yield curve approaches unity at all incident energies (see Fig. 4c).

\section{CONCLUSIONS}

The studies described in this paper have demonstrated that pulsed electron methods provide an effective way to measure the "intrinsic" electron emission properties of uncharged insulators. They have also been shown to provide a sensitive tool to explore the effects of accumulated charge from incident electron beams on the electron emission properties of insulators. Indeed, electron emission properties have been shown to be very sensitive to charge accumulation, showing pronounced effects after as little as $<3 \mathrm{fC} / \mathrm{mm}^{2}$ of incident charge. The effect of internal charge accumulation has been quantitatively observed on the secondary yield. Distinct behaviors have also been observed in yield decay curves between the crossover energies, due to the build up of positive charge.

Simple modifications have been made to a physics-based (Chung-Everhart) model for the spectral emission of secondary electrons and coupled with existing models for the internal charge distribution resulting from electron emission for insulators. This union has provided an expression that correctly describes the behavior of the secondary yield as positive potential accumulates on the material surface. We have also developed an expression for the yield decay curves, which measure the total yield modifications as a function of cumulative incident charge. Studies have begun to simultaneously measure the electron yield and surface potential to more fully test our model.

The expressions for fitting the yield decay curves allows us to reconstruct yield as a function of both incident energy and specific incident pulse fluences. We have found strong evidence that the dual-peaks observed in the traditional low fluence pulse yield measurement are the result of positive surface charging. This method provides us with a way to measure the uncharged yield in insulators with high resistivity and high yield that would not otherwise be possible.

Two important questions are raised by this study that will be pursed in future work. First, we note that some previous studies of the electron yield curves of high yield, high resistivity insulators using very high fluence beams (many orders of magnitude higher than our study) have measured yield curves similar to our "intrinsic" yield curves, rather than double peak or unity yield curves characteristic of a highly charged sample[28], [29]. Often such studies use highly focused beams from AES or SEM systems, with beam diameters $<1 \mu \mathrm{m}$ and fluences $10^{4}-10^{6}$ times higher than our studies. We speculate that the local sample resistivity of the insulator may be greatly reduced due to radiation induced conductivity (RIC) leading to charge dissipation within the sample. We also note that RIC persists for some time after the beam is turned off, so that this explanation could also be applicable to pulsed or rastered probe beams. For a Kapton HN sample, a rastered $1 \mathrm{keV}$ incident energy with an estimated penetration depth of $30 \mathrm{~nm}$ at a 10 A-mm-2 effective beam density produces an average absorbed dose rate of $10^{5}$ Gy and a radiation induced conductivity of $10^{-10} \Omega^{-1}-\mathrm{cm}^{-1}$ (with $10^{-2} \mathrm{~s}$ decay time), $10^{9}$ times larger than the zero dose rate dark current conductivity. In Kapton HN radiation induced conductivity persisted above $10 \%$ of the equilibrium dose rate value for $>100 \mathrm{~s}$ [30]. This explanation is closely related to a study of Green and Dennison of the measurements of resistivity by the charge storage method for an intense, rastered proton beam [31].

The other question that arises is whether our studies of the "intrinsic" and charged insulator yields with low fluence beams are relevant to models of insulators charging and yields in the space environment. One might argue that all insulators will quickly charge to the point that all yields will be unity. Alternately, one might argue that only "intrinsic" yields are relevant for very low space environments fluxes and that only RIC-enhanced yields like those measured with high fluence test apparatus are relevant for high flux space environments. However, it appears (at least for certain high yield, high resistivity materials used in the construction of spacecraft) that typical ambient space environment fluxes may well produce charging conditions similar to those in our low fluence yield measurements. The answers to both of these questions are open issues that certainly merit continued study.

\section{ACKNOWLEDGMENTS}

The authors would like to thank J. Abbott, J. Cazaux and O. Jbara for discussions and insight into the problem. We acknowledge useful modeling of the surface potential by Dan Arnfeld.

\section{REFERENCES}

[1] JR Dennison, R.C. Hoffmann, and J. Abbott, "Triggering Threshold Spacecraft Charging with Changes in Electron Emission from Materials,” Paper AIAA-2007-1098, Proceedings of the 45th American Institute of Aeronautics and Astronomics Meeting on Aerospace Sciences, Reno, NV, 2007

[2] Chung, M. S., and T. E. Everhart, "Simple calculation of energy distribution of low-energy secondary electrons emitted from metals under electron bombardment,” J. Appl. Phys. 45 (2), 707-709 (1974).

[3] Chung, M.S.,"Improved calculations of secondary electron energy distributions of metals," J. Appl. Phys. 46 (2), 465-466 (1975)

[4] Cazaux, J., "Some considerations on the secondary electron emission $\delta$, form e- irradiated insulators,” J. Appl. Phys. 85 (2), 1137-1147 (1999).

[5] Cazaux, J., "A new model of dependence: secondary electron emission yield on primary electron energy for application to polymers", J. Phys. D: Appl. Phys., 38, 2433-2441, (2005).

[6] Meyza, X., Goeuriot, D., Guerret-Piecout, C., Treheux, D., Fitting, H.J., "Secondary Electron Emission and Self-consistent Charge Transport and Storage in Bulk Insulators: Application to Alumina,” J. Appl. Phys., 94, 5384- 5392, (2003).

[7] Sébastien Clerc J.R. Dennison and Clint Thomson, "Importance of Accurate Computation of Secondary Electron Emission for Modeling Spacecraft Charging," Proceedings of the 9th Spacecraft Charging Technology Conference, (EPOCHAL TSUKUBA, 2005). 
[8] J.R. Dennison, C.D. Thomson, J. Kite, V. Zavyalov, J. Corbridge, "Materials Characterization at USU: Facilities and Knowledgebase of Electronic Properties Applicable to Spacecraft Materials," Proc. 8th Spacecraft Charging Tech. Conf., (Huntsville, AL, USA), 2004.

[9] Nickles, N. E., "The Role of Bandgap in the Secondary Electron Emission of Small Bandgap Semiconductors: Studies of Graphitic Carbon”, Ph.D. dissertation, Utah State University, Logan, UT 2002.

[10] C.D. Thomson, "Measurements of Secondary Electron Emission Properties of Insulators," Ph.D. dissertation, Utah State University, Logan, UT, 2004.

[11] Alec Sim, J.R. Dennison and Clint Thomson, "Effects Of Incident Electron Fluence And Energy On The Electron Yield Curves And Emission Spectra Of Dielectrics,” Bull. Am. Phys. Soc. 50(1) Part II, (2005).

[12] N. Nickles, R.E. Davies, J.R. Dennison, "Applications of Secondary Electron Energy- and Angular-Distributions to Spacecraft Charging," Proc. of the 8th Spacecraft Charging Tech. Conf., (AFRL Sc. Center, Hanscom AFB, MA, USA), 2000.

[13] L. Reimer, Scanning Electron Microscopy. Physics of Image Formation and Microanalysis, New York, USA: Springer-Verlag, 119-121, 1985.

[14] Sternglass, E. J., "Secondary electron emission and atomic shell structure,” Phys. Rev. 80, 925-926 (1950); Sternglass, E. J., An Experimental Investigation of Electron Back-Scattered and Secondary Electron Emission From Solids, Ph.D. Dissertation, Cornell University, 1953.

[15] Young, J. R., "Penetration of electrons and ions in aluminum," J. Appl. Phys. 27 (1), 1-4 (1956).

[16] A. Melchinger, S. Hofmann, "Dynamic double layer model: Description of time dependent charging phenomena in insulators under electron beam irradiation,” J. Appl. Phys. 78, 6224-32, 2003.

[17] Cazaux, J., private communications, 2005.

[18] Cazaux, J., K. H. Kim, O. Jbara, and G. Salace, "Charging effects of MgO under electron bombardment and nonohmic behavior of the induced specimen current,” J. Appl. Phys. 70 (2), 960-965 (1991); Cazaux, J., "Some considerations on the secondary electron emission $\delta$ from e- irradiated insulators,” J. Appl. Phys. 85, 1137-1147 (1999).

[19] Cazeaux, J., "Charging in Scanning Electron Microscopy 'from Inside and Outside'," Scanning, page\# 26, 2004.

[20] Miyake, H., Y. Tanaka, and T. Takada, "Characteristic of charge accumulation in glass materials under electron beam irradiation," Proceedings of the 8th Spacecraft Charging Technology Conference, Huntsville, AL, (2003).

[21] Osawa, N., S. Takahashi, Y. Tanaka, T. Takada, R. Watanabe, N. Tomita, V. Griseri, L. Levy, and C. Laurent, "Measurement of bulk charge in dielectric materials irradiated by electron beam in vacuum environment," Proceedings of the 8th Spacecraft Charging Technology Conference, Huntsville, AL, (2003).

[22] Usui, Y., T. Sakai, M. Ishikawa, T. Isono, Y. Tanaka, T. Takada, R. Watanabe, N. Tomita, and Y. Murooka, "Measurement of charge distribution in electron beam irradiated PMMA using electro-optical effect," Proceedings of the 8th Spacecraft Charging Technology Conference, Huntsville, AL, (2003).

[23] Tatsuo Takada, "Pulse Acoustic Technology for Measurement of Charge Distribution in Dielectric Materials for Spacecraft," Proceedings of the 9th Spacecraft Charging Technology Conference, (EPOCHAL TSUKUBA, 2005).

[24] Davies, R. E., Measurement of Angle-resolved Secondary Electron Spectra, PhD Dissertation, Utah State University, 1999.

[25] Chang, W. Y, J. R. Dennison, J. Kite, and R. E. Davies, "Effects of evolving surface contamination on spacecraft charging," Proceedings of the 38th American Institute of Aeronautics and Astronomics Meeting on Aerospace Sciences, Reno, NV (2000b).

[26] C.D. Thomson, V. Zavyalov, J.R. Dennison, "Instrumentation for studies of electron emission and charging from insulators," Proc. of the 8th Spacecraft Charging Tech. Conf., (Huntsville, AL, USA), 2004.

[27] C.D. Thomson, V. Zavyalov, J.R. Dennison, J. Corbridge, "Electron Emission Properties of Insulator Materials Pertinent to the International Space Station,” Proc. of the 8th Spacecraft Charging Tech. Conf., (Huntsville, AL, USA), 2004.

[28] N.R. Whetten, "Cleavage in High Vacuum of Alkali Halide Single Crystals-Secondary Electron Emission”, J.Appl. Phys. 35, 3279 (1964)

[29] P.H. Dawson, "Secondary Electron Emission Yields of Some Ceramics,” J. Appl. Phys. 35, 3644 (1966).

[30] JR Dennison, Jodie Gillespie, Joshua Hodges, RC Hoffmann, J Abbott, Alan W. Hunt and Randy Spalding, "Radiation Induced Conductivity of
Highly-Insulating Spacecraft Materials," Proceedings of the 10th Spacecraft Charging and Technology Conference, (Biarritz, France, June 18-21, 2007).

[31] N.W. Green, and JR Dennison, "Deep Dielectric Charging of Spacecraft Polymers by Energetic Protons,” Proc. of the 10th Spacecraft Charging Tech. Conf., (Biarritz, France), 2007
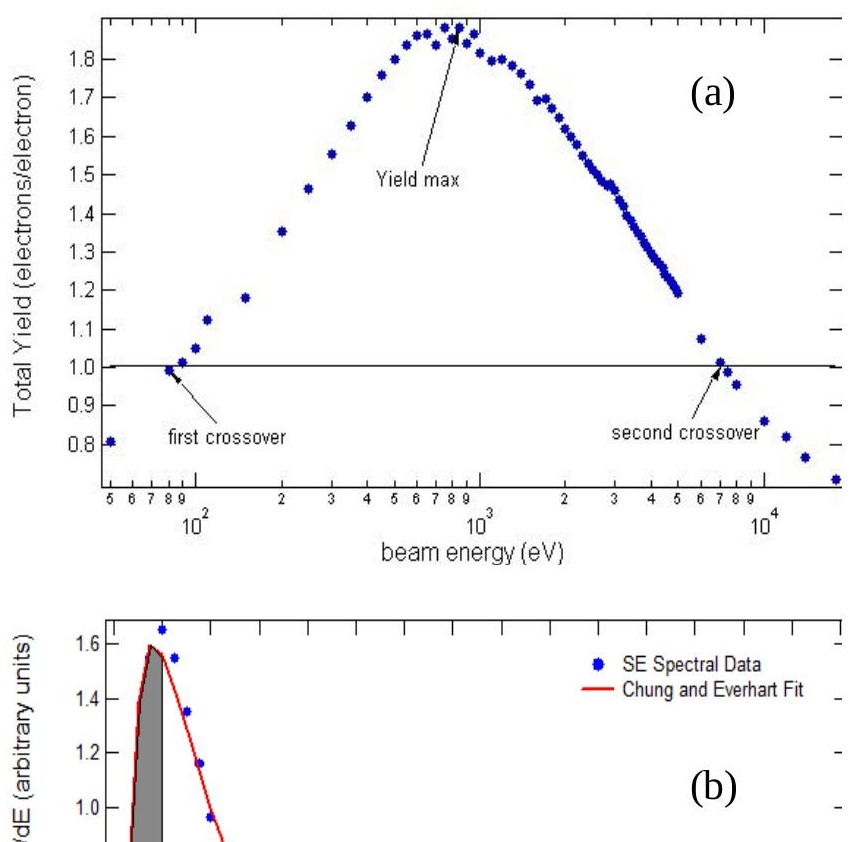


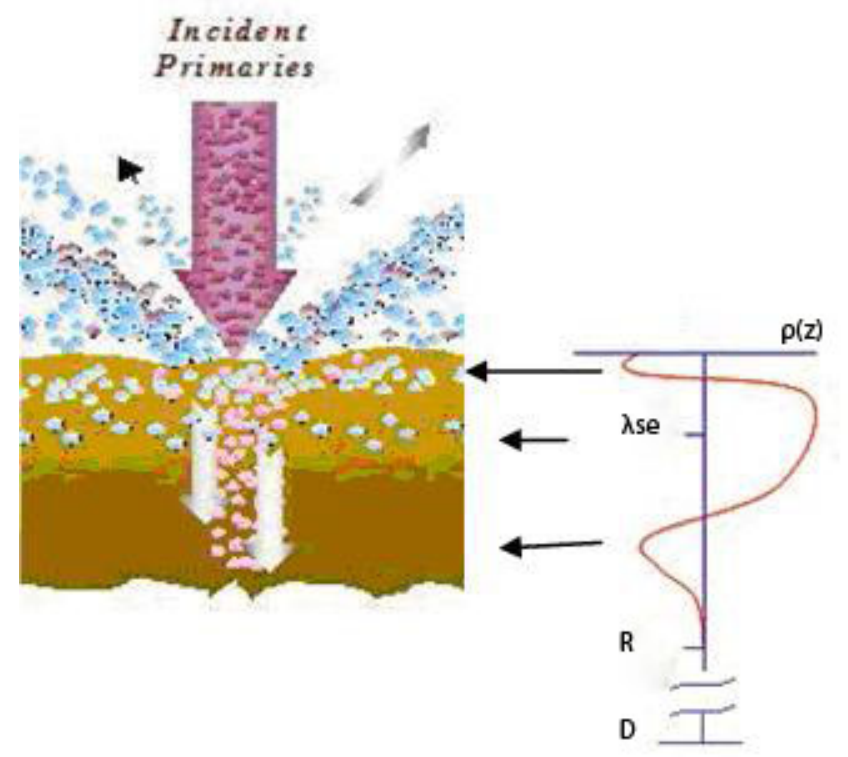

Fig. 2. (Left) Standard models of electron emission divide the process into three stages: production, SE transport and escape. Primary electrons (PE) of energy $E_{o}$ impinge on the surface and penetrate up to a depth $R$ Secondary electrons (SE) are produced within the material and some are
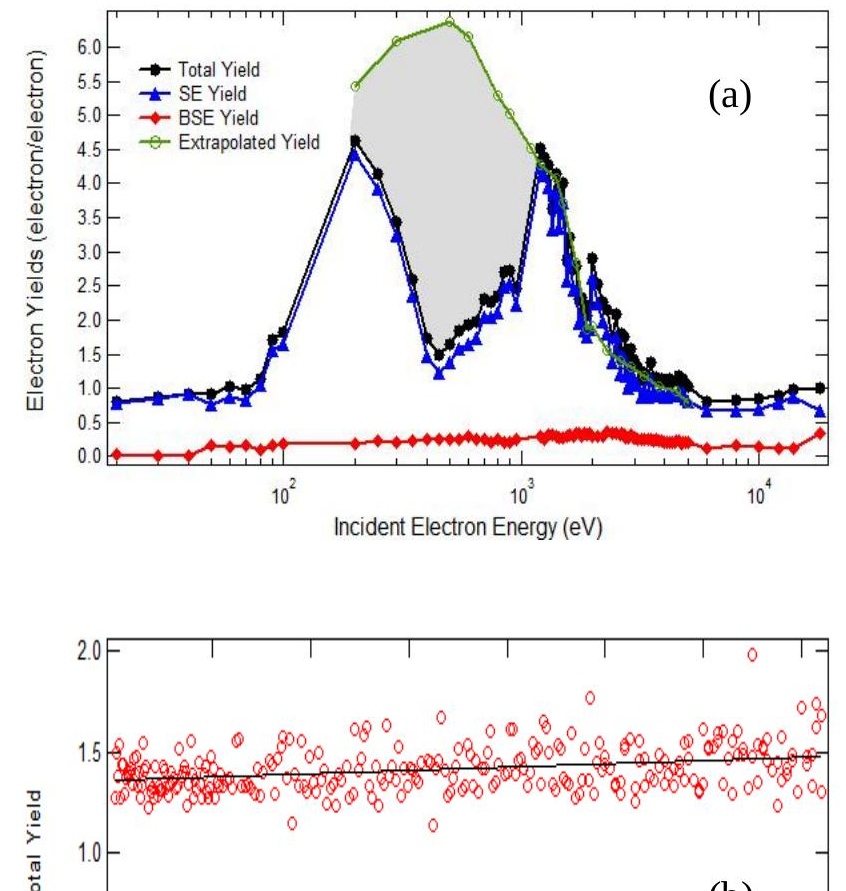
